hep-th/0610116

MCTP-06-21

UMDEPP 06-050

NSF-KITP-06-70

\title{
Gauged $(2,2)$ Sigma Models and Generalized Kähler Geometry
}

\author{
Willie Merrell ${ }^{1}$, Leopoldo A. Pando Zayas ${ }^{2}$ and Diana Vaman ${ }^{2}$ \\ ${ }^{1}$ Department of Physics \\ University of Maryland \\ College Park, MD 20472 \\ ${ }^{2}$ Michigan Center for Theoretical Physics \\ Randall Laboratory of Physics, The University of Michigan \\ Ann Arbor, MI 48109
}

\begin{abstract}
We gauge the $(2,2)$ supersymmetric non-linear sigma model whose target space has bihermitian structure $\left(g, B, J_{ \pm}\right)$with noncommuting complex structures. The bihermitian geometry is realized by a sigma model which is written in terms of $(2,2)$ semi-chiral superfields. We discuss the moment map, from the perspective of the gauged sigma model action and from the integrability condition for a Hamiltonian vector field. We show that for a concrete example, the $S U(2) \times U(1)$ WZNW model, as well as for the sigma models with almost product structure, the moment map can be used together with the corresponding Killing vector to form an element of $T \oplus T^{*}$ which lies in the eigenbundle of the generalized almost complex structure. Lastly, we discuss T-duality at the level of a $(2,2)$ sigma model involving semi-chiral superfields and present an explicit example.
\end{abstract}




\section{Introduction}

Superfield representations and geometry: The connection between geometry and supersymmetry has been an evolving and recurring theme since the early days of supersymmetry. The earliest example was the connection between Kähler geometry and supersymmetric non linear sigma models introduced in a seminal paper by Zumino [1] who considered the conditions for existence of $\mathcal{N}=(2,2)$ on two-dimensional nonlinear sigma models. This notion was further developed by Álvarez-Gaumé and Freedman who showed that further extensions of supersymmetry to $\mathcal{N}=(4,4)$ required the sigma model metric to be hyperkähler [2].

Other important geometric structures were understood in this context including the moment map as well as symplectic and Kähler reductions. Many of these structures were devoloped independently in the mathematical and physics literature. The use of a Legendre transform and a symplectic quotient in the study of hyperkähler geometry arose from their use in supersymmetric sigma models [3-5]. In the context of hyperkähler geometry a comprehensive review was presented in [6].

An important step in understanding the general structure behind geometry and supersymmetry was taken in [7] which presented a classification of the geometries consistent with extended supersymmetry paying particular attention to the type of superfield representations involved. Perhaps the clearest example of the connection between geometry and the superfield representations arose out of the study of twodimensional $\mathcal{N}=(2,2)$ supersymmetric models discovered by Gates, Hull, and Roček [8]. This work introduced twisted chiral superfields on the supersymmetry side and bihermitian geometry on the mathematical side. Subsequently, Buscher, Lindström, and Roček [9] expanded in this direction. These results showed that the underlying element in the relation between the amount of supersymmetry and different versions of complex geometry is to a large extent determined by the superfield representations involved.

Generalized complex geometry and $(2,2)$ supersymmetry: What would complex geometry look like if instead of considering structures associated with the tangent bundle $T$ one considers structures associated with the direct product of the tangent and cotangent bundle $T \oplus T^{*}$ ? This question has recently been posed by Hitchin leading to the formulation of generalized complex geometry [10]. Generalized complex geometry naturally contains complex, symplectic, Kähler and bihermitian geometries as particular 
cases. A fairly complete account can be found in Gualtieri's thesis [11]. Several geometric concepts, like the moment map, reduction of generalized complex and generalized Kähler geometries, and others are currently being developed [12-17]. A number of works have sought to clarify the connection of generalized complex geometry to supersymmetry. A very interesting analysis presented in [18] showed how the integrability conditions of the generalized complex structure could be understood, at the nonlinear sigma model level, as the conditions for a manifestly $(1,1)$ supersymmetric model to be $(2,2)$ supersymmetric. In the language of representations it has also become increasingly evident that semi-chiral superfields play a central role [18-22].

The role of semi-chiral superfields and our work: The mathematical literature seems to allow for some ambiguities in the definition of some of the geometric structures involved. In particular, several groups have proposed definitions of the reduction of generalized complex geometry and moment map [14-16,23]. On the physics side, these concepts are related to gauging some of the symmetries of the nonlinear sigma model. The above situation motivates us to approach these concepts from the physics point of view. We also believe that understanding nonlinear sigma models and the gauging of some of the symmetries in the most general context is an important problem in physics. We have heavily relied on previous works that addressed such questions in the context of complex and Kähler geometry and partially in the case of bihermitian geometry [24-27]. A concept that does not seem to be intrinsic to the mathematical literature but that we will investigate, following in part the work of Roček and Verlinde [28], is T-duality in the presence of semi-chiral superfields. More generally, the goal of this paper is to lay the groundwork for exploring the connection between generalized complex geometry and supersymmetry in terms of gauged nonlinear sigma models with superfields in the semi-chiral representation.

The paper is organized as follows. In section 2 we discuss the gauging of the sigma model with semi-chiral superfields under the simplifying assumption that the Kähler potential is invariant under the action of the $U(1)$ symmetry. We reduce the action to $(1,1)$ superspace and find, via comparison with [24], the moment map and the one form required for gauging a sigma model with $B$ field. We analyze the example of the $S U(2) \otimes U(1)$ WZNW model and verify the identifications we have made for the moment map and one form and point out an ambiguity which arises in the presence of semi-chiral representations. We conclude section 2 with a description of a gauging based on the prepotential. In section 3 we briefly review the mathematical literature 
on Hamiltonian action and moment map for generalized complex geometry. We also compare the mathematical definition with the physical definition of the moment map and explicitly discus the example of the $S U(2) \otimes U(1)$ WZNW model. In section 4 we address T-duality in $(2,2)$ superspace. We first describe T-duality in case of chiral and twisted chiral superfields following the formalism used in [28], but with a different gauge fixing procedure. Next, we use this gauge fixing procedure to describe T duality in the case when semi-chiral superfields are used. We finish by working out the $4 \mathrm{~d}$ flat space example. In section 5 we draw some conclusions and point out some interesting open questions.

\section{Gauging a $(2,2)$ sigma model and the moment map}

One property of $(2,2)$ superspace is that the sigma model is given entirely in terms of the Kähler potential:

$$
\mathcal{S}=\int d^{2} x D^{2} \bar{D}^{2} K(\{\Phi, \bar{\Phi}\})
$$

Geometric quantities associated with the sigma model, such as the target space metric and B field, can be obtained in explicit form by performing a reduction to $(1,1)$ superspace. The specific properties of the sigma model target space geometry follow from the specific choice of $N=2$ superfields $\{\Phi\}$. If $\{\Phi\}$ is a set of chiral superfields $\bar{D}_{ \pm} \Phi=0$, or twisted chiral superfields $D_{+} \Phi=\bar{D}_{-} \Phi=0$, then the associated target space geometry is Kähler. If both chiral and twisted chiral superfields are found among $\{\Phi\}$, then the corresponding geometry goes by the name of "bihermitian geometry" with almost product structure. This particular geometry is characterized by two commuting almost complex structures $J_{ \pm}, J_{ \pm}^{2}=-1$, which are integrable and covariantly constant with respect to affine connections with torsion, a metric that is bihermitian with respect to both complex structures $J_{ \pm}^{t} g J_{ \pm}=g$, and a $B$-field. As a consequence of the fact that $\left[J_{+}, J_{-}\right]=0$, the metric acquires a block diagonal form, inducing a natural decomposition of the tangent space, along the chiral, respectively, twisted chiral components. It is the feature of having two commuting complex structures that distinguishes the almost product structure geometry among the class of bihermitian geometries. Lastly, if the set of superfields which determine the Kähler potential contains a more general $(2,2)$ semi-chiral superfield, constrained only by a single superspace covariant derivative $D_{+} \Phi=0$, or $D_{-} \Phi=0$, then the sigma model target space is bihermitian, with non-commuting complex structures. Recently, it has 
been shown [11] that the projection of the generalized Kähler geometry onto the tangent bundle yields the bihermitian data $\left(g, J_{ \pm}, B\right)$ found by Gates, Hull and Roček [8] when investigating non-linear sigma models with $(2,2)$ supersymmetry.

If the sigma model target space has an isometry group, then a generic Killing vector can be decomposed in a basis of the Killing vectors $k_{A}$ which generate the Lie algebra of the isometry group

$$
\xi=\xi^{A} k_{A}=\xi^{A} k_{A}^{i} \partial_{i}, \quad\left[k_{A}, k_{B}\right]=f_{A B}^{C} k_{C}, \quad \mathcal{L}_{\xi} g=0 .
$$

The infinitesimal transformation of the sigma-model fields is given by

$$
\delta \phi^{i}=\epsilon^{A} k_{A}^{i}
$$

where $\epsilon^{A}$ are rigid infinitesimal parameters. For a sigma model with isometries, there are additional geometric data. These follow from the integrability conditions associated with the additional requirements that the action of the Killing vector leave invariant not just the metric, but the field strength of the B-field $H$, and the symplectic forms $\omega_{ \pm}=g J_{ \pm}$:

$$
\mathcal{L}_{\xi} H=0, \quad \mathcal{L}_{\xi} \omega_{ \pm}=0
$$

From the condition that $H$ is invariant, it follows that

$$
\mathcal{L}_{\xi} H=d i_{\xi} H+i_{\xi} d H=d i_{\xi} H=0 .
$$

Since the two-form $i_{\xi} H$ is closed, locally it can be written as

$$
i_{\xi_{A}} H=d u_{A}
$$

where the one-form $u$ is determined up to an exact, Lie-algebra valued one-form. The ambiguity in $u$ can be fixed, up to $U(1)$ factors in the Lie algebra, by requiring that it is equivariant $\mathcal{L}_{A} u_{B}=f_{A B}{ }^{C} u_{C}$.

Besides this one form $u$, the other geometric datum associated with the existence of an isometry group is the moment map (also known as Killing potential). From the condition that the symplectic form is invariant under $\xi$, and from $d \omega_{ \pm}\left(J_{ \pm} X, J_{ \pm} Y, J_{ \pm} Z\right)=$ $\pm H(X, Y, Z)$, it follows that $\omega_{ \pm} \xi \mp J_{ \pm}^{T} u$ is closed. Therefore, locally one finds

$$
d \mu_{ \pm}=\omega_{ \pm} \xi \mp J_{ \pm}^{T} u
$$

where $\mu_{ \pm}$are the moment maps. This expression is the generalization for a manifold with torsion of the integrability condition for a Hamiltonian vector field $\xi$. Since $\xi$ is also Killing, it follows that $\mathcal{L}_{\xi} J_{ \pm}=0$. 
The relevance of these two quantities, the one-form $u$ and the moment map $\mu$, becomes obvious when constructing the gauged sigma model, by promoting the rigid (global) isometries 2.3 to local ones. This is accompanied, in the usual manner, by introducing a compensating connection (gauge potential) $\partial_{\mu} \phi^{i} \longrightarrow \nabla_{\mu} \phi=\partial_{\mu} \phi^{i}+A_{\mu}^{A} k_{A}^{i}$, which transforms as $\delta A_{\mu}^{A}=\partial_{\mu} \epsilon^{A}+f_{A B}{ }^{C} A_{\mu}^{B} \epsilon^{C}$. For $(1,0)$ or $(1,1)$ supersymmetric sigma models, the bosonic gauge connection becomes part of a corresponding $(1,0)$ or $(1,1)$ super Yang-Mills multiplet. Promoting the partial derivatives to gauge covariant derivatives is not enough in the presence of a $B$-field $[24,27,29]$. New terms, which depend on the $u$ one-form and the moment map, must be added to the sigma model action. For a bosonic, $(1,0)$ or $(1,1)$ supersymmetric sigma-model, adding only $u$ dependent terms suffices:

$$
\mathcal{S}=\int d^{2} x d^{2} \theta\left(g_{i j} \nabla_{+} \phi^{i} \nabla_{-} \phi^{j}+B_{i j} D_{+} \phi^{j} D_{-} \phi^{j}-2 u_{i A} A_{(+}^{A} D_{-} \phi^{i}+A_{+}^{A} A_{-}^{B} c_{[A B]}\right),
$$

where $D_{ \pm}$are flat superspace covariant derivatives and $\nabla_{ \pm}$are superspace gauge covariant derivatives, while $c_{[A B]}=k_{[A}^{i} u_{i B]}$.

When the sigma model has additional supersymmetries, then the gauged sigma model action acquires new terms, which are moment map dependent. The additional supersymmetries, which at the level of $N=1$ superspace are nonlinearly realized, are of the form

$$
\delta \phi^{i}=\epsilon J^{i}{ }_{j} D_{+} \phi^{j}
$$

The supersymmetry algebra requires that the $(1,1) \operatorname{tensor}(\mathrm{s}) J^{i}{ }_{j}$ be identified with the almost complex structure(s). The gauged $(2,2)$ sigma model action typically contains a term

$$
\delta \mathcal{S}=\int d^{2} x d^{2} \theta S \mu
$$

where $\mu$ is the moment map, and $S$ is a super-curvature that appears in the $(2,2)$ superalgebra (more precisely in the super-commutators $\left\{\nabla_{+}, \bar{\nabla}_{-}\right\}$).

The above formulae show that the moment map and the one-form $u$ are necessary ingredients to gauge the sigma model. Intuitively, the one-form $u$ is needed to gauge the sigma model with $B$ field and the moment map is needed to gauge a sigma model with extended supersymmetry.

Alternatively, one could choose instead to remain at the level of $(2,2)$ superspace and perform the gauging there, without ever descending onto the $(1,1)$ superspace. In the process, the Kähler potential of the ungauged sigma model $K(\{\Phi\})$ acquires a new term, which is also moment-map dependent [24,25]. 
We shall be interested in gauging $(2,2)$ sigma models whose target space has a bihermitian structure, with non-commuting complex structures. The natural starting point for us is the $N=2$ superspace formulation of a sigma-model written in terms of $(2,2)$ semi-chiral superfields:

$$
\begin{aligned}
& \text { left chiral: } \quad \bar{D}_{+} X=0, \\
& \text { right antichiral: } \quad D_{-} Y=0 .
\end{aligned}
$$

We begin by making the observation that the following transformations are consistent with the contraints 1 on $X$ and $Y$.

$$
\begin{aligned}
& X \rightarrow(A+B) X+C+D, \\
& Y \rightarrow(F+G) Y+W+Z,
\end{aligned}
$$

where $A, C$ are chiral superfields, $B, D$ are twisted anti chiral superfields, $F, W$ are anti chiral, and $G, Z$ are twisted chiral. When these transformations correspond to gauge transformations they can be properly accounted for using both the chiral and twisted chiral vector multiplets.

For simplicity we will consider only the gauge transformations where the semichiral superfields are multiplied and shifted by chiral and anti-chiral superfields 2 . In this paper we shall follow two complementary approaches to constructing the gauged action in $(2,2)$ superspace. The first method involves descending at the level of $(1,1)$ superspace by following the usual route of substituting the Grassmann integration by differentiation $\int d \theta d \bar{\theta} \rightarrow D \bar{D}$, and by the subsequent replacement of the ordinary superspace covariant derivatives by gauge covariant derivatives $D \bar{D} \rightarrow \nabla \bar{\nabla}$. This is equivalent to gauging by minimal coupling, if the Kähler potential is invariant under the action of the isometry generators. The second method [25] uses the prepotential of the gauge multiplet $V$ explicitly in the Kähler potential to restore the invariance of the action under local transformations.

For simplicity we restrict ourselves to $U(1)$ isometries. As such we can go to a coordinate system where the isometry is realized by a shift of some coordinate. This implies that the Kähler potential $K(X, \bar{X}, Y, \bar{Y})$ will be independent of a certain linear combination of the left and right semi-chiral superfields. For example, for

$$
K=K(X+\bar{X}, Y+\bar{Y}, X+Y)
$$

\footnotetext{
${ }^{1}$ This is not the most general set of transformations consistent with the constraints on $X$ and $Y$. However, these are the only transformations relevant to our considerations.

${ }^{2}$ We thank S. Gates for various clarifications on this point.
} 
we can immediately read off the Killing vector associated with the isometry. In this case it takes the form

$$
\xi=i \frac{\partial}{\partial X}-i \frac{\partial}{\partial \bar{X}}-i \frac{\partial}{\partial Y}+i \frac{\partial}{\partial \bar{Y}}
$$

From (2.12) we see that this is an example of a Kähler potential, with a $U(1)$ isometry which can be gauged using only the (un-twisted) $(2,2)$ super Yang-Mills multiplet.

\subsection{Gauging and the reduction to $(1,1)$ superspace}

Let us consider the first of the two approaches to gauging which we have outlined before. Since we are interested in extracting the geometric data (including those associated with isometries) from the sigma model, and these are most easily seen in the language of $(1,1)$ superspace, here we describe the bridge from $(2,2)$ to $(1,1)$ superspace, following [8] closely.

We begin by recording the $(2,2)$ gauge covariant supersymmetry algebra for the (un-twisted) super Yang-Mills multiplet $\left(A_{\alpha}^{A}, \bar{A}_{\alpha}^{A}, A_{\neq}^{A}, A_{=}^{A}\right)$ :

$$
\begin{aligned}
& {\left[\nabla_{\alpha}, \nabla_{\beta}\right\}=0} \\
& {\left[\nabla_{\alpha}, \bar{\nabla}_{\beta}\right\}=2 i\left(\gamma^{c}\right)_{\alpha \beta} \nabla_{c}+2 g\left[C_{\alpha \beta} S-i\left(\gamma^{3}\right)_{\alpha \beta} P\right] \xi,} \\
& {\left[\nabla_{\alpha}, \nabla_{b}\right\}=\lambda\left(\gamma_{b}\right)_{\alpha}{ }^{\beta} \bar{W}_{\beta} \xi,} \\
& {\left[\nabla_{a}, \nabla_{b}\right\}=-i \lambda \epsilon_{a b} \mathcal{W} \xi,}
\end{aligned}
$$

where the bosonic two-dimensional indices are $a, b, c=\{\neq,=\}$, and the Grassmann odd two-dimensional spinor indices are $\alpha= \pm$. The skew-symmetric tensor $C_{\alpha \beta}$ is used for raising and lowering indices in superspace. Having in mind the gauging of a certain isometry of a $(2,2)$ sigma model, we used the Killing vector $\xi$ to denote the couplings of the sigma model superfields to the $(2,2)$ super Yang-Mills multiplet.

Also, following from the Bianchi identities, one has the following set of constraints

$$
\begin{aligned}
\nabla_{\alpha} S & =-i \bar{W}_{\alpha}, \quad \nabla_{\alpha} P=-\left(\gamma^{3}\right)_{\alpha}{ }^{\beta} \bar{W}_{\beta}, \\
\nabla_{\alpha} \bar{W}_{\beta} & =0, \quad \nabla_{\alpha} d=\left(\gamma^{c}\right)_{\alpha}^{\beta} \nabla_{c} \bar{W}_{\beta}, \\
\nabla_{\alpha} W_{\beta} & =i C_{\alpha \beta} d-\left(\gamma^{3}\right)_{\alpha \beta} \mathcal{W}+\left(\gamma^{a}\right)_{\alpha \beta} \nabla_{a} S-i\left(\gamma^{3} \gamma^{a}\right)_{\alpha \beta} \nabla_{a} P .
\end{aligned}
$$

According to our previous discussion on gauging methods, we take our first step towards constructing the gauged $(2,2)$ sigma model begin by making the substitution

$$
\int d^{2} \bar{\theta} d^{2} \theta=\frac{1}{8}\left[\nabla^{\alpha} \nabla_{\alpha} \bar{\nabla}^{\beta} \bar{\nabla}_{\beta}+\bar{\nabla}^{\beta} \bar{\nabla}_{\beta} \nabla^{\alpha} \nabla_{\alpha}\right]
$$


where we have used the conventions of [30].

In order to reduce the action written in $(2,2)$ superspace to $(1,1)$ superspace we need to express the $(2,2)$ gauge covariant derivatives in terms of two copies of the $(1,1)$ derivatives:

$$
\hat{\nabla}_{\alpha}=\frac{1}{\sqrt{2}}\left(\nabla_{\alpha}+\bar{\nabla}_{\alpha}\right), \quad \tilde{\nabla}_{\alpha}=\frac{i}{\sqrt{2}}\left(\nabla_{\alpha}-\bar{\nabla}_{\alpha}\right) .
$$

The $(1,1)$ derivatives satisfy the following algebra:

$$
\begin{aligned}
{\left[\hat{\nabla}_{\alpha}, \hat{\nabla}_{\beta}\right\} } & =2 i\left(\gamma^{c}\right)_{\alpha \beta} \nabla_{c}-2 i \lambda\left(\gamma^{3}\right)_{\alpha \beta} P \xi \\
{\left[\hat{\nabla}_{\alpha}, \nabla_{b}\right\} } & =\frac{\lambda}{\sqrt{2}}\left(\gamma_{b}\right)_{\alpha}{ }^{\beta} \hat{W}_{\beta} \xi, \\
{\left[\tilde{\nabla}_{\alpha}, \tilde{\nabla}_{\beta}\right\} } & =2 i\left(\gamma^{c}\right)_{\alpha \beta} \nabla_{c}-2 i \lambda\left(\gamma^{3}\right)_{\alpha \beta} P \xi, \\
{\left[\tilde{\nabla}_{\alpha}, \nabla_{b}\right\} } & =\frac{\lambda}{\sqrt{2}}\left(\gamma_{b}\right)_{\alpha}{ }^{\beta} \tilde{W}_{\beta} \xi, \\
{\left[\hat{\nabla}_{\alpha}, \tilde{\nabla}_{\beta}\right\} } & =-2 i \lambda C_{\alpha \beta} S \xi,
\end{aligned}
$$

where $\tilde{W}_{\beta}=\frac{i}{\sqrt{2}}\left(\bar{W}_{\beta}-W_{\beta}\right)$ and $\hat{W}_{\beta}=\frac{1}{\sqrt{2}}\left(\bar{W}_{\beta}+W_{\beta}\right)$.

Next we consider the measure of the $(2,2)$ action (2.17) and we rewrite it in terms of the $(1,1)$ derivatives

$$
\hat{\nabla}^{\alpha} \hat{\nabla}_{\alpha} \tilde{\nabla}^{\beta} \tilde{\nabla}_{\beta}=2 \nabla^{\alpha} \nabla_{\alpha} \bar{\nabla}^{\beta} \bar{\nabla}_{\beta}+2 \bar{\nabla}^{\beta} \bar{\nabla}_{\beta} \nabla^{\alpha} \nabla_{\alpha}+(\ldots) \xi+\text { total derivative }
$$

Therefore $\hat{\nabla}^{\alpha} \hat{\nabla}_{\alpha} \tilde{\nabla}^{\beta} \tilde{\nabla}_{\beta}$ and $2 \nabla^{\alpha} \nabla_{\alpha} \bar{\nabla}^{\beta} \bar{\nabla}_{\beta}+2 \bar{\nabla}^{\beta} \bar{\nabla}_{\beta} \nabla^{\alpha} \nabla_{\alpha}$ are equivalent when acting on a potential which is invariant under the isometry, that is, satisfies $\xi K=0$.

Reducing the $(2,2)$ Lagrangian amounts to evaluating

$$
L=\int d^{2} \bar{\theta} d^{2} \theta K=\frac{1}{4} \hat{\nabla}^{2} \tilde{\nabla}^{2} K(X, \bar{X}, Y, \bar{Y}),
$$

where the semi-chiral superfields $X, \bar{X}, Y, \bar{Y}$ obey (2.11), but with the ordinary superspace derivative $D_{ \pm}$replaces by the gauge-covariant derivatives (2.15). Then, using the relation

$$
\tilde{\nabla}^{\alpha} \tilde{\nabla}_{\alpha}=-2 i \tilde{\nabla}_{+} \tilde{\nabla}_{-}-2 i \lambda P \xi,
$$

we only need to evaluate $\tilde{\nabla}_{+} \tilde{\nabla}_{-} K$. Additionally, we must decompose the $(2,2)$ left and right semi-chiral superfields into $(1,1)$ superfields

$$
\varphi=X\left|, \quad \Psi=\tilde{\nabla}_{-} X\right|, \quad \chi=Y\left|, \quad \Upsilon=\tilde{\nabla}_{+} Y\right| .
$$

We end up with:

$$
\tilde{\nabla}_{+} \tilde{\nabla}_{-} K=\hat{\nabla}_{+} \varphi^{I} m_{I I^{\prime}} \hat{\nabla}_{-} \chi^{I^{\prime}}+\Upsilon_{+}^{I^{\prime}} n_{I^{\prime} I} \Psi_{-}^{I}+\Psi_{-}^{I}\left(2 \omega_{I J} \hat{\nabla}_{+} \varphi^{J}+i p_{I I^{\prime}} \hat{\nabla}_{+} \chi^{I^{\prime}}\right)
$$




$$
\begin{aligned}
& +\Upsilon_{+}^{I^{\prime}}\left(2 \omega_{I^{\prime} J^{\prime}} \hat{\nabla}_{-} \chi^{J^{\prime}}-i q_{I^{\prime} I} \hat{\nabla}_{-} \varphi^{I}\right)-2 i \lambda S K_{i^{\prime}} \xi^{i^{\prime}}+2 i g S K_{\bar{i}^{\prime}} \xi^{\bar{i}^{\prime}} \\
& +2 i \lambda(S+i P) K_{i} \xi^{i}-2 i \lambda(S-i P) K_{\bar{i}} \xi^{\bar{i}} \\
= & \hat{\nabla}_{+} \Phi^{T} \cdot E \cdot \hat{\nabla}_{-} \Phi+S_{+I} u^{I I^{\prime}} S_{-I^{\prime}}-2 i \lambda S K_{i^{\prime}} \xi^{i^{\prime}}+2 i \lambda S K_{\bar{i}^{\prime}} \xi^{\bar{i}^{\prime}} \\
& +2 i \lambda(S+i P) K_{i} \xi^{i}-2 i \lambda(S-i P) K_{\bar{i}} \xi^{\bar{i}}
\end{aligned}
$$

where we have used the notation $K_{i}=\partial_{\varphi^{i}} K, K_{i^{\prime}}=\partial_{\chi^{i^{\prime}}} K$ and that the U(1) Killing vector is $\xi=\xi^{i} \partial_{\varphi^{i}}+\xi^{i^{\prime}} \partial_{\chi^{i^{\prime}}}+\xi^{\bar{i}} \partial_{\bar{\varphi} \bar{i}}+\xi^{\bar{i}^{\prime}} \partial_{\bar{\chi}^{i^{\prime}}}$. The index $I$ is a collective index: $I=\{i, \bar{i}\}$, and $\Phi=\{\phi, \bar{\phi}, \chi, \bar{\chi}\}$. The matrices $m, n, \omega, p, q$, expressed in terms of the second order derivatives of the Kähler potential, are the same as in [19]. Also, analogous to [19]

$$
\begin{aligned}
S_{+I} u^{I I^{\prime}} & =\Upsilon_{+}^{I^{\prime}}-2 u^{I I^{\prime}} \omega_{I J} \hat{\nabla}_{+} \varphi^{J}-i u^{I I^{\prime}} P_{I J^{\prime}} \hat{\nabla}_{+} \chi^{J^{\prime}} \\
u^{I I^{\prime}} S_{-I^{\prime}} & =\Psi_{-}^{A}+2 u^{I I^{\prime}} \omega_{I^{\prime} J^{\prime}} \hat{\nabla}_{-} \chi^{J^{\prime}}-i u^{I I^{\prime}} q_{I^{\prime} J} \hat{\nabla}_{-} \varphi^{B} \\
E & =g+B=\left(\begin{array}{cc}
2 i \omega u q & m-4 \omega u \omega^{\prime} \\
p^{t} u q & 2 i p^{t} u \omega^{\prime}
\end{array}\right) .
\end{aligned}
$$

At a first glance it appears that we have an asymmetric coupling of the field strength $P$ between the fields $\varphi^{I}$ and $\chi^{I^{\prime}}$. However, this is just an artifact of our choice in evaluating the covariant derivatives. Note that

$$
\xi K(\varphi, \chi)=0 \rightarrow K_{i} \xi^{i}+K_{\bar{i}} \xi^{\bar{i}}+K_{i^{\prime}} \xi^{i^{\prime}}+K_{\bar{i}^{\prime}} \xi^{\bar{i}^{\prime}}=0 .
$$

This means that the reduced Lagrangian is given by

$$
\begin{aligned}
L= & \hat{\nabla}^{\alpha} \hat{\nabla}_{\alpha}\left(\hat{\nabla}_{+} \Phi^{T} \cdot E \cdot \hat{\nabla}_{-} \Phi+S_{+I} u^{I I^{\prime}} S_{-I^{\prime}}\right. \\
& +2 i \lambda\left(S+\frac{i}{2} P\right) K_{i} \xi^{i}-2 i \lambda\left(S-\frac{i}{2} P\right) K_{\bar{i}} \xi^{\bar{i}} \\
& \left.-2 i g\left(S+\frac{i}{2} P\right) K_{i^{\prime}} \xi^{i^{\prime}}+2 i g\left(S-\frac{i}{2} P\right) K_{\bar{i}^{\prime}} \xi^{\bar{i}^{\prime}}\right) \\
= & \hat{\nabla}^{\alpha} \hat{\nabla}_{\alpha}\left(\hat{\nabla}_{+} \Phi^{T} \cdot(g+B) \cdot \hat{\nabla}_{-} \Phi+S_{+I} u^{I I^{\prime}} S_{-I^{\prime}}\right. \\
& +2 i \lambda S\left(K_{i} \xi^{i}-K_{\bar{i}} \xi^{\bar{i}}-K_{i^{\prime}} \xi^{i^{\prime}}+K_{\bar{i}^{\prime}} \xi^{\bar{i}^{\prime}}\right) \\
& \left.-\lambda P\left(K_{i} \xi^{i}+K_{\bar{i}} \xi^{\bar{i}}-K_{i^{\prime}} \xi^{i^{\prime}}-K_{\bar{i}^{\prime}} \xi^{\bar{i}^{\prime}}\right)\right) .
\end{aligned}
$$

This is the gauged sigma model we were after, and it is one of our main results.

To understand the various terms that appear in (2.27), it is useful to compare this action with (2.8), given that both actions represent gauged sigma models with manifest $(1,1)$ supersymmetry. This explains the obvious common elements $\hat{\nabla}^{\alpha} \Phi^{T} \cdot g \cdot \hat{\nabla}_{\alpha} \Phi+$ $\hat{D}^{\alpha} \Phi^{T} \cdot B \cdot \hat{D}_{\alpha} \Phi$. The gauging of the $B$-field terms is done in (2.8) by including the $u$ dependent terms. How about in our case? First, we notice that since we have assumed 
that $\xi K=0$, in other words that the minimal coupling prescription will suffice, this is indeed what the gauged sigma model Lagrangian (2.27) reflects. The extra terms required for the gauging of the $B$-field terms can be combined into $\left.i_{\xi} B \cdot \hat{D}_{(-} \Phi A_{+}\right)$. As a consequence of the assumption $\xi K=0$, we find that $\mathcal{L}_{\xi} B=0$. This is a stronger condition than $\mathcal{L}_{\xi} H=0$, and it implies the latter. Since $\mathcal{L}_{\xi} B=0$, we find

$$
u=-i_{\xi} B+d \sigma,
$$

where $d \sigma$ is an exact one-form invariant under the action of the isometry group. This is exactly what is required to match the minimal coupling of the $B$-field terms against the $u$-terms in (2.8). The $c_{A B}$ terms in (2.8) vanish in the case of a $U(1)$ gauging. Otherwise, they, too, could be recognized in the minimal coupling gauging of (2.8).

We shall see that the ambiguity in defining $u$, namely the exact one-form $d \sigma$, is reflected in (2.27) in the term which multiplies the field strength $P$. The expression $-\lambda d\left(K_{I} \xi^{I}-K_{I^{\prime}} \xi^{I^{\prime}}\right)$ is $d(\sigma)$. We verify that it is invariant under the $\mathrm{U}(1)$ action:

$$
\begin{aligned}
\mathcal{L}_{\xi} d \sigma & =d\left(i_{\xi} d \sigma\right)=d\left(\left(\xi^{I} \partial_{I}+\xi^{I^{\prime}} \partial_{I^{\prime}}\right)\left(\xi^{J} \partial_{J}-\xi^{J^{\prime}} \partial_{J^{\prime}}\right) K\right)= \\
& =2 d\left(\left(\xi^{I} \partial_{I}+\xi^{I^{\prime}} \partial_{I^{\prime}}\right) \xi^{I} \partial_{J} K\right)=2 d\left(\left(-\xi^{I} \partial_{I} \xi^{I^{\prime}} \partial_{I^{\prime}}+\xi^{I^{\prime}} \partial_{I^{\prime}} \xi^{I} \partial_{I}\right) K\right)=0
\end{aligned}
$$

where in the last step we used that we can go to a coordinate system where the $\mathrm{U}(1)$ action is realized by a shift of some coordinate, which implies $\left[\xi^{I} \partial_{I}, \xi^{I^{\prime}} \partial_{I^{\prime}}\right]=0$.

The remaining terms in (2.27), such as those dependent on the auxiliary superfields $S_{ \pm}$and which have no counterpart in (2.8), are present because our starting point was a $(2,2)$ supersymmetric action with off-shell $(2,2)$ superfields. Lastly, we recognize in the terms proportional to the superfield strength $S$, a linear combination of the moment maps. Their presence is required to insure the invariance of the gauged sigma model action. While the expression proportional to $\mathrm{S}$ in (2.27) is not immediately relatable to the moment map given in (2.7), it does have a form similar to that given in $[31,24,32]$ for the moment map. There the moment map is identified as the imaginary part of the holomorphic transformation of the Kähler potential under the action of the Killing vector.

Thus we conclude with the identifications:

$$
\begin{aligned}
& \text { Moment map } \sim K_{i} \xi^{i}-K_{\bar{i}} \xi^{\bar{i}}-K_{i^{\prime}} \xi^{i^{\prime}}+K_{\bar{i}^{\prime}} \xi^{\bar{i}^{\prime}} \\
& \sigma \sim K_{a} \xi^{i}+K_{\bar{i}} \xi^{\bar{i}}-K_{i^{\prime}} \xi^{i^{\prime}}-K_{\bar{i}^{\prime}} \xi^{\bar{i}^{\prime}} .
\end{aligned}
$$


These identifications, and especially the rapport between (2.30) and (2.7), will be verified in the next section.

\subsection{An example: the $S U(2) \times U(1)$ WZNW model}

In this section we apply our previous construction of a $(2,2)$ gauged sigma model to a concrete example: the $S U(2) \times U(1)$ WZNW model. The $(2,2)$ supersymmetric $S U(2) \times U(1)$ WZNW sigma model was first formulated in terms of semi-chiral superfields in [33]. These authors discovered non-commuting complex structures on $S U(2) \times U(1)$ and constructed a duality functional that does not change the geometry. However, this duality functional allows to map between two seemingly different descriptions, one for $S U(2) \times U(1)$ described in terms of chiral and twisted chiral superfields and the another description in terms of semi-chiral superfields. The explicit form of the Kähler potential was given in [34,35]. A discussion on the various dual descriptions which can be obtained by means of a Legendre transform can be found in [35]. The $S U(2) \times U(1)$ Kähler potential is

$$
K=-(\bar{\phi}+\eta)(\phi+\bar{\eta})+\frac{1}{2}(\bar{\eta}+\eta)^{2}-2 \int^{\bar{\eta}+\eta} d x \ln \left(1+e^{x / 2}\right)
$$

where $\bar{D}_{+} \phi=D_{-} \eta=0$. Because $K=K(\bar{\phi}+\eta, \phi+\bar{\eta}, \eta+\bar{\eta})$ we cannot directly gauge the theory, using only the coupling with the (un-twisted) $(2,2)$ super Yang-Mills multiplet. However, there is an easy remedy to this problem, namely we shall use a dual description, found via a Legendre transform [35]:

$$
K(r, \bar{r}, \eta, \bar{\eta})=K(\phi, \bar{\phi}, \eta, \bar{\eta})-r \phi-\bar{r} \phi
$$

where $r$ is semi-chiral, $\bar{D}_{+} r=0$, and $\phi$ is unconstrained. By integrating over $r$, we recover the previous Kähler potential. On the other hand, by integrating over $\phi$, that is eliminating it from its equation of motion, we find a Kähler potential $K=K(r+$ $\eta, \bar{r}+\bar{\eta}, \eta+\bar{\eta}$ ) (up to terms that represent a generalized Kähler transform $\frac{1}{2} \eta^{2}+\frac{1}{2} \bar{\eta}^{2}$ ). This is an example of a "duality without isometry" [35], where the Kähler potential of a semi-chiral superfield sigma model can be mapped via Legendre transforms into four different, but equivalent expressions, all involving only semi-chiral superfields.

The new form taken by the $S U(2) \times U(1)$ Kähler potential

$$
\tilde{K}=(\bar{r}+\bar{\eta})(r+\eta)-2 \int^{\bar{\eta}+\eta} d x \ln \left(1+e^{x / 2}\right)
$$


indicates that the $U(1)$ isometry is realized by the transformations

$$
r \rightarrow r+i \epsilon, \quad \eta \rightarrow \eta+\overline{(i \epsilon)}
$$

where $\epsilon$ is a constant real parameter. However, when promoting this symmetry to a local one, according to our previous discussion, $\epsilon$ is to be interpreted as a chiral superfield, and $\bar{\epsilon}$ as an anti-chiral superfield.

The Kähler potential is left invariant under the action of the $(2,2)$ Killing vector

$$
\xi=i \frac{\partial}{\partial r}-i \frac{\partial}{\partial \bar{r}}-i \frac{\partial}{\partial \eta}+i \frac{\partial}{\partial \bar{\eta}}
$$

From (2.25) we can now calculate the B field, its field strength and their contractions with the Killing vector:

$$
\begin{aligned}
& B=(1-2 f)(d r \wedge d \bar{\eta}+d \bar{r} \wedge d \eta) \\
& \mathrm{i}_{\xi} B=i(1-2 f) d \bar{\eta}-i(1-2 f) d \eta+i(1-2 f) d \bar{r}-i(1-2 f) d r \\
& H=d B=2\left(\frac{\partial f}{\partial \eta} d r-\frac{\partial f}{\partial \bar{\eta}} d \bar{r}\right) \wedge d \eta \wedge d \bar{\eta} \\
& \mathrm{i}_{\xi} H=d(2 i f[-d r+d \bar{r}-d \eta+d \bar{\eta}])=d u,
\end{aligned}
$$

where

$$
f=f(\bar{\eta}+\eta)=\frac{\exp \left[\frac{1}{2}(\bar{\eta}+\eta)\right]}{1+\exp \left[\frac{1}{2}(\bar{\eta}+\eta)\right]} .
$$

We also find that $\mathcal{L}_{\xi} B=0$, in accord to the expectation that the gauging is done via minimal coupling $[27,29]$. As discussed before, it implies that $u=-\mathrm{i}_{\xi} B+d \sigma$ where $d \sigma$ is an exact one-form, invariant under the action of the Killing vector. As to the term proportional to $P$ in (2.27) we find that is equal to $2 i \lambda \sigma$, where $d \sigma=d(\bar{r}-r+\bar{\eta}-\eta)$. Indeed, this one-form satisfies the condition $i_{\xi} d \sigma=0$.

Next, we show how the term proportional to $S$ corresponds to the moment map.

\subsubsection{The Moment Map}

Here we verify that the term proportional to the super-curvature $S$ in (2.27)

$$
i\left(K_{r}-K_{\bar{r}}-K_{\eta}+K_{\bar{\eta}}\right)=2 i\left[r+\bar{r}+\eta+\bar{\eta}-2 \ln \left(1+\exp \left(\frac{\eta+\bar{\eta}}{2}\right)\right)\right] \equiv M
$$


is a certain linear combination of the two moment maps of the bihermitian geometry. We recall their definition

$$
g_{i j} \xi^{j} \pm u_{i}=I_{ \pm i}^{j} \partial_{j} \mu_{ \pm}
$$

Before we consider (2.40) we must first address the ambiguity in the expression for the one form $u$. The one-form $u$ is defined only up to an exact one form that satisfies $\mathcal{L}_{\xi} d \sigma=0: u=2 i f[-d r+d \bar{r}-d \eta+d \bar{\eta}]+d i\left(C_{r} r+C_{\bar{r}} \bar{r}+C_{\eta} \eta+C_{\bar{\eta}} \bar{\eta}\right)$ with $C_{r, \bar{r}, \eta, \bar{\eta}}$ constants, constrained only by $C_{r}-C_{\bar{r}}-C_{\eta}+C_{\bar{\eta}}=0$. However, our previous considerations have eliminated most of the freedom in $d \sigma$, given that, from the gauged action we have identified $C_{r}=-1, C_{\bar{r}}=1, C_{\eta}=-1, C_{\bar{\eta}}=1$. Armed with the concrete expressions of the moment maps we find the following relationship with $M$ :

$$
M=-\left(\mu_{+}+\mu_{-}\right)
$$

\subsection{Alternative gauging procedure: the prepotential}

In section 2.1 we gauged the sigma model by replacing the Grassmann integration measure with gauge supercovariant derivatives and thus reducing the $(2,2)$ action to a gauged action with $(1,1)$ manifest supersymmetry. Here we take the alternative approach of using the gauge prepotential superfield $V$ to arrive at a gauge-invariant Kähler potential. This procedure is done in $(2,2)$ superspace, and all supersymmetries remain manifest. Therefore this gauging method has the advantage of facilitating the discussion of duality functionals, which we will address in the next section.

In simple cases, the gauging is done by adding the prepotential $V$ to the appropriate combination of superfields in the Kähler potential: for the example $K=K(X+\bar{X}, Y+$ $\bar{Y}, X+Y)$, the global symmetry is promoted to a local one via the substitution

$$
K(X+\bar{X}, Y+\bar{Y}, X+Y) \rightarrow K(X+\bar{X}+V, Y+\bar{Y}+V, X+Y+V)
$$

if the gauging is done using the (un-twisted) $(2,2)$ super Yang-Mills prepotential, in other words, if the gauge parameter is a chiral superfield. On the other hand, if the gauge parameter is a twisted chiral superfield, then we must use the gauge prepotential associated with a twisted $(2,2)$ super Yang-Mills multiplet $V_{t}$. For example, we could gauge $K=K(X+\bar{X}, Y+\bar{Y}, X+\bar{Y})$ by

$$
K(X+\bar{X}, Y+\bar{Y}, X+\bar{Y}) \rightarrow K\left(X+\bar{X}+V_{t}, Y+\bar{Y}+V_{t}, X+\bar{Y}+V_{t}\right)
$$


For concreteness we continue to address only the gauging done using the coupling to the (un-twisted) $(2,2)$ super Yang-Mills multiplet. In general, the isometry transformations of a given superfield are given by:

$$
X \rightarrow e^{i \epsilon \xi} X \Rightarrow \bar{X} \rightarrow e^{-i \bar{\epsilon} \xi} \bar{X}
$$

where $\xi$ denotes the isometry generator and $\epsilon$ is a real valued constant parameter. When promoting this global symmetry to a local one, the gauge parameter $\epsilon$ becomes a chiral superfield, and $\bar{\epsilon}$ an anti-chiral superfield. The invariance is restored by introducing the gauge prepotential superfield $V$, transforming as

$$
V \rightarrow V+i(\bar{\epsilon}-\epsilon)
$$

We include $V$ through the replacement:

$$
\bar{X} \rightarrow e^{i V \xi} \bar{X}
$$

Now $\bar{X}$ transforms in the same way as in the global case and thus the invariance has been restored.

Although we have used the whole Killing vector $\xi$ in constructing the field that transforms properly (2.46), to be more specific, it is only the part of the Killing vector that induces a transformation with the anti-chiral gauge parameter which contributes to this definition. In the example that we gave, $K=K(X+\bar{X}, Y+\bar{Y}, X+Y), X, \bar{Y}$ transform with a chiral gauge parameter, and $\bar{X}, Y$, with an anti-chiral parameter. The Killing vector will generally factorize $\xi=\xi_{c}+\xi_{\bar{c}}$ such that $\xi_{c}$ and $\bar{\xi}_{c}$ induce a chiral parameter, respectively an anti-chiral parameter gauge transformation. In the $S U(2) \otimes U(1)$ example we have $\xi_{\bar{c}}=-i \frac{\partial}{\partial \bar{r}}-i \frac{\partial}{\partial \eta}$.

Therefore we define

$$
\tilde{X}=e^{L} \bar{X}, \quad L=i V \xi_{\bar{c}} .
$$

The new field, $\tilde{X}$, transforms under the gauge transformation in the exact same way as $\bar{X}$ did under the global isometry. Therefore by replacing $\bar{X}$ in the Kähler potential by $\tilde{X}$ we insure that the transformation of the Kähler potential under the local transformation is the same as for the global isometry, namely it is a generalized Kähler transformation. Of course, the other semi-chiral superfield $Y$ undergoes a similar treatment:

$$
\tilde{Y}=e^{L} Y
$$


If the Kähler potential remains invariant under the action of the Killing vector i.e. $\xi K(X, \bar{X}, Y, \bar{Y})=0$, the minimal coupling perscription is given by replacing $\bar{X}$ with $\tilde{X}$ and $Y$ with $\tilde{Y}$. Specifically, the gauged $(2,2)$ Lagrangian is given by the replacement

$$
K(X, \bar{X}, Y, \bar{Y}) \rightarrow K(X, \tilde{X}, \tilde{Y}, \bar{Y})
$$

At this point we can use the relation $K(X, \tilde{X}, \tilde{Y}, \bar{Y})=e^{L} K(X, \bar{X}, Y, \bar{Y})$ to rewrite the Lagrangian as

$$
\begin{aligned}
K(X, \tilde{X}, \tilde{Y}, \bar{Y}) & =e^{L} K(X, \bar{X}, Y, \bar{Y})=K(X, \bar{X}, Y, \bar{Y})+\frac{e^{L}-1}{L} L K \\
& =K(X, \bar{X}, Y, \bar{Y})+\frac{e^{L}-1}{L} V M,
\end{aligned}
$$

where in $M=i \xi_{\bar{c}} K$ we recognized the same object which we have identified from the gauged $(1,1)$ action as the moment map (3.4).

Next, we address the case of a Kähler potential which under the action of the isomtery generator transforms with terms that take the form of generalized Kähler transformations

$$
\xi K=f(X)+\bar{f}(\bar{X})+g(Y)+\bar{g}(\bar{Y}) .
$$

The trick is to introduce new coordinates and add them to the Kähler potential in such a way that the new Kähler potential is invariant under the transformation generated by the new Killing vector. Specifically we introduce $\alpha, \beta$ with $\bar{D}_{+} \alpha=D_{-} \beta=0$. We construct the new Kähler potential and Killing vector

$$
\begin{aligned}
& K^{\prime}(X, \bar{X}, Y, \bar{Y}, \alpha, \bar{\alpha}, \beta, \bar{\beta})=K(X, \bar{X}, Y, \bar{Y})-\alpha-\bar{\alpha}-\beta-\bar{\beta} \\
& \xi^{\prime}=\xi+f(X) \frac{\partial}{\partial \alpha}+\bar{f}(\bar{X}) \frac{\partial}{\partial \bar{\alpha}}+g(Y) \frac{\partial}{\partial \beta}+\bar{g}(\bar{Y}) \frac{\partial}{\partial \bar{\beta}}
\end{aligned}
$$

Now the new Kähler potential $K^{\prime}$ is invariant under the new Killing vector $\mathcal{L}_{\xi^{\prime}} K^{\prime}=0$ and we can proceed as before. We replace all fields which transform with the parameter $\bar{\epsilon}$ with the combination which transforms with the field $\epsilon$ by using $e^{L^{\prime}}$ where $L^{\prime}=i V \xi_{\bar{c}}^{\prime}$. Next we define the tilde versions of $\bar{X}, Y, \bar{\alpha}, \beta$ as follows

$$
\tilde{X}=e^{L^{\prime}} \bar{X}, \quad \tilde{Y}=e^{L^{\prime}} Y, \quad \tilde{\alpha}=e^{L^{\prime}} \bar{\alpha} \quad \tilde{\beta}=e^{L^{\prime}} \beta .
$$

The gauged Lagrangian is obtained by the same substitution as before. Finally we get

$$
K^{\prime}(X, \tilde{X}, \tilde{Y}, \bar{Y}, \alpha, \tilde{\alpha}, \tilde{\beta}, \bar{\beta})=K(X, \tilde{X}, \tilde{Y}, \bar{Y})-\alpha-\tilde{\alpha}-\tilde{\beta}-\bar{\beta}
$$




$$
\begin{aligned}
& =e^{L} K(X, \bar{X}, Y, \bar{Y})-i \frac{e^{L}-1}{L} V(\bar{f}(\bar{X})+g(Y)) \\
& =K(X, \bar{X}, Y, \bar{Y})+\frac{e^{L}-1}{L}(L K-i V \bar{f}(\bar{X})-i V g(Y)) \\
& =K(X, \bar{X}, Y, \bar{Y})+\frac{e^{L}-1}{L} V M .
\end{aligned}
$$

\section{Eigenspaces of Generalized Complex Structures}

\subsection{Hamiltonian action and moment map in the mathematical literature}

In the context of generalized complex geoemtry, the origin of subsequent definitions of the Hamiltonian action can be found in Gualtieri's thesis [11] where it was shown that certain infinitesimal symmetries preserving the generalized complex structure $\mathcal{J}$ can be extended to second order.

Intuitively, given a Hamiltonian action on a generalized complex manifold, the moment map is a quantity that is constant along the action of the group elements. More formal definitions of moment map were given, for example, in [14-16,36]; in [16] $\mathrm{Hu}$ considered the Hamiltonian group globally. For concreteness here we will explore one of the definitions put forward by Lin and Tolman [14] in the simplest setting without $H$-twisting, namely, definition 3.4:

Let a compact Lie group $G$ with Lie algebra $g$ act on a manifold $M$, preserving a generalized complex structure $\mathcal{J}$. Let $L \in T \oplus T^{*}$ denote the $\sqrt{-1}$-eigenbundle of $\mathcal{J}$. A generalized moment map is a smooth function $\mu: M \rightarrow g^{*}$ so that

(i) $\xi_{M}-\sqrt{-1} d \mu^{\xi}$ lies in $L$ for all $\xi \in g$, where $\xi_{M}$ denotes the induced vector field on $M$.

(ii) $\mu$ is equivariant.

In subsequent works, the definition of Hamiltonian action was generalized to include the $H$-twisted case $[15,16]$. In [17], the authors arrived at a definition of moment map in terms of the action of a Lie algebra on a Courant algebroid.

In what follows we will explore the particular definition cited above, and compare it with the expressions that we gave for the moment map in the previous sections. We leave for a future publication the issue of the equivalence of the various definitions given in the math literature, and their relationship with the physical point of view advocated in this paper, via the gauging of the $(2,2)$ sigma model. 


\subsection{Generalized Kahler geometry and the eigenvalue problem}

In a series of papers $[19,20]$ the authors established that chiral, twisted chiral, and semi-chiral superfields are the most generic off-shell multiplets for $\mathcal{N}=(2,2)$ supersymmetric non-linear sigma models. The use of these $(2,2)$ multiplets yields generalized Kähler geometries.

To practically use the above definition of moment map in the case of Kähler geometry we recall that, according to Gualtieri (see Chapter 6 in [11]), the generalized complex structures of the generalized Kähler geometry take the following expressions:

$$
\mathcal{J}_{1 / 2}=\frac{1}{2}\left(\begin{array}{ll}
1 & 0 \\
B & 1
\end{array}\right)\left(\begin{array}{cc}
J_{+} \pm J_{-} & -\left(\omega_{+}^{-1} \mp \omega_{-}^{-1}\right) \\
\omega_{+} \mp \omega_{-} & -\left(J_{+}^{t} \pm J_{-}^{t}\right)
\end{array}\right)\left(\begin{array}{cc}
1 & 0 \\
-B & 1
\end{array}\right)
$$

where $g$ is a Kähler metric, which is bihermitian with respect to both complex structures $J_{ \pm}$, while $B$ is a 2 -form field. We leave a discussion about its relationship with the $B$-field of the sigma model for section 3.4.

First, we shall derive the conditions for a generic element of $(\xi, \pm i d \mu) \in T \oplus T^{*}$ to be an eigenvector of the generalized complex structures. By identifying $\xi \in T$ with a Killing vector, we solve for the one form $d \mu \in T^{*}$. Next, after verifying that $d \mu$ is an exact one-form, we shall compare it with the the moment map and enquire whether these expressions are compatible. We discuss two concrete settings: the almost product structure spaces, with their commuting complex structures, and as an example of bihermitian geometry we turn to the $S U(2) \times U(1)$ WZNW sigma model.

We begin with some formal statements. The condition that an element of $T \oplus T^{*}$ lies in the eigenbundle of $\mathcal{J}_{1}$ is

$$
\mathcal{J}_{1}\left(\begin{array}{c}
\xi \\
i c d \mu
\end{array}\right)=a i\left(\begin{array}{c}
\xi \\
i c d \mu
\end{array}\right)
$$

where $c= \pm 1, a= \pm 1$. After a bit of massaging, we find that this eigenvalue problem is equivalent to the following linear homogeneous equation system ${ }^{3}$

$$
\left(J_{+}-a i\right)(\Gamma-\xi)=0
$$

\footnotetext{
${ }^{3}$ For the eigenvalue problem associated with the other generalized almost complex structure $\mathcal{J}_{2}$, we find a similar linear homogeneous system:

$$
\left(J_{+}-a i\right)(\Gamma-\xi)=0, \quad\left(J_{-}+a i\right)(\Gamma+\xi)=0 .
$$
}




$$
\left(J_{-}-a i\right)(\Gamma+\xi)=0,
$$

where

$$
\Gamma=G^{-1}(B \xi-i c d \mu)
$$

Then, by solving (3.3) we find $\xi$ and $\Gamma$. The number of independent solutions is equal to the number of zero eigenvalues of $J_{ \pm}-a i$. After identifying $\xi$ with a certain Killing vector, we generically find a corresponding $\Gamma$. This allows us to solve for $\mu$ :

$$
d \mu=i c(G \Gamma-B \xi)
$$

To test the compatibility between this expression and the moment map (2.7), in the next sections we explore two concrete examples of bihermitian geometry.

\subsection{Specialization to spaces with almost product structure}

In the case of a space with almost product structure, which is realized by a $(2,2)$ sigma model written in terms of chiral and twisted chiral superfields [8], we may choose to work in a coordinate system where the two commuting complex structures are diagonal:

$$
J_{+}=\left(\begin{array}{cc}
J_{1} & 0 \\
0 & J_{2}
\end{array}\right) \quad J_{-}=\left(\begin{array}{cc}
J_{1} & 0 \\
0 & -J_{2}
\end{array}\right) .
$$

In the same coordinate system, the metric and $B$-field are also block-diagonal:

$$
g=\left(\begin{array}{cc}
g_{1} & 0 \\
0 & g_{2}
\end{array}\right) \quad B=\left(\begin{array}{cc}
0 & b \\
-b^{t} & 0
\end{array}\right) .
$$

The expressions taken by $g, B, J_{+}$, and $J_{-}$suggest that we should consider a similar decomposition for $\xi, \Gamma$ and $d \mu$. Specifically,

$$
\xi=\left(\begin{array}{l}
\xi_{1} \\
\xi_{2}
\end{array}\right) \quad \Gamma=\left(\begin{array}{c}
\Gamma_{1} \\
\Gamma_{2}
\end{array}\right) \quad d \mu=\left(\begin{array}{c}
d \mu_{1} \\
d \mu_{2}
\end{array}\right) .
$$

Under this decomposition $\Gamma_{1,2}, \xi_{1,2}$ are solutions to (3.3):

$$
\begin{aligned}
& \left(J_{1}-a i\right) \Gamma_{1}=\left(J_{1}-a i\right) \xi_{1}=0 \\
& a i \Gamma_{2}=-J_{2} \xi_{2} .
\end{aligned}
$$


and (3.5) becomes

$$
\begin{aligned}
& d \mu_{1}=i c g_{1} \Gamma_{1}-i c b \xi_{2} \\
& d \mu_{2}=i c g_{2} \Gamma_{2}+i c b^{t} \xi_{1},
\end{aligned}
$$

How does this compare with the moment maps which are given by $d \mu_{ \pm}=\omega_{ \pm} \xi \mp J_{ \pm}^{T} u$ ? When we specialize to the case where the Lie derivative of $B$ with respect to $\xi$ vanishes, $\mathcal{L}_{\xi} B=0$, we can use that $u=-B \xi+d \sigma$. Taking the appropriate linear combinations that match up most closely with the generalized complex structures we define

$$
d \tilde{M}=\frac{1}{2}\left(d \mu_{+}+d \mu_{-}\right), \quad d \hat{M}=\frac{1}{2}\left(d \mu_{+}-d \mu_{-}\right)
$$

where

$$
\left(\begin{array}{c}
d \tilde{M}_{1} \\
d \tilde{M}_{2}
\end{array}\right)=\left(\begin{array}{c}
\omega_{1} \xi_{1} \\
-J_{2}^{t} b^{t} \xi_{1}
\end{array}\right), \quad\left(\begin{array}{c}
d \hat{M}_{1} \\
d \hat{M}_{2}
\end{array}\right)=\left(\begin{array}{c}
-J_{1}^{t} b \xi_{2} \\
\omega_{2} \xi_{2}
\end{array}\right) .
$$

The matching between (3.10) and (3.12) can be done using either $d \tilde{M}$ or $d \hat{M}$. For concreteness, we choose to match (3.10) against $d \hat{M}$. This is done provided that $\Gamma_{1}=\xi_{1}=0$. The condition $\xi_{1}=0$ is automatically satisfied for almost product structure geometries, where $J_{1,2}$ are both diagonal. This is so because the requirement that $\xi$ is holomorphic (i.e. it leaves invariant the complex structures) implies that that either $\xi_{1}$ or $\xi_{2}$ vanish [24]. Next to complete the matching of (3.12) and (3.10) we need $\Gamma_{2}= \pm i J_{2} \xi_{2}$, but is exactly the expression of $\Gamma_{2}$ which we get from (3.9).

Now that we have verified the compatibility of two moment map definitions, (3.5) and (2.7), for the almost product structure geometry, we want to investigate their compatibility in a more generic case of bihermitian geometry. Since the complex structures do not commute in this case, it is difficult to analyze what happens in general. However we can consider the concrete $S U(2) \otimes U(1)$ example and see how things work out there.

\subsection{The $S U(2) \times U(1)$ example}

In this case, the non-commuting complex structures, read off from the supersymmetry transformations of the non-linear sigma model [19,33], are:

$$
J_{+}=\left(\begin{array}{cccc}
i & 0 & 0 & 0 \\
0 & -i & 0 & 0 \\
-2 i & 0 & -i & 0 \\
0 & 2 i & 0 & i
\end{array}\right) \quad J_{-}=\left(\begin{array}{cccc}
i & 0 & 2 i(1-f) & 0 \\
0 & -i & 0 & -2 i(1-f) \\
0 & 0 & -i & 0 \\
0 & 0 & 0 & i
\end{array}\right)
$$


where $f=f(\eta+\bar{\eta})$. The $\mathrm{U}(1)$ Killing vector is $\xi=(i,-i,-i, i)$. The $B$-field was given in (2.37), and the metric takes the form

$$
g=\left(\begin{array}{cccc}
0 & 2 & 0 & 2(1-f) \\
2 & 0 & 2(1-f) & 0 \\
0 & 2(1-f) & 0 & 2(1-f) \\
2(1-f) & 0 & 2(1-f) & 0
\end{array}\right) .
$$

The moment map $d \mu_{+}=\omega_{+} \xi-J_{+}^{T} u$ reads

$$
\begin{aligned}
d \mu_{+} & =(-2 f,-2 f, 0,0)-(-2 f,-2 f,-2 f,-2 f)-\left(i C_{r}-2 i C_{\eta},-i C_{\bar{r}}+2 i C_{\bar{\eta}},-i C_{\eta}, i C_{\bar{\eta}}\right) \\
& =\left(i C_{r}-2 i C_{\eta},-i C_{\bar{r}}+2 i C_{\bar{\eta}}, 2 f-i C_{\eta}, 2 f+i C_{\bar{\eta}}\right) .
\end{aligned}
$$

wheare the last term on the first line represents the ambiguity in $u, J_{+}^{T} d \sigma$. The constants $C_{r, \bar{r}, \eta, \bar{\eta}}$ satisfy the constraint $C_{r}-C_{\bar{r}}-C_{\eta}+C_{\bar{\eta}}=0$.

We find that the solution to (3.3), corresponding to a $+i$ eigenvector, $(a=1)$, is given by $\left(\xi, \Gamma_{1, \pm}\right)$, where $\xi=(i,-i,-i, i)$ and

$$
\Gamma_{1,+}=\left(-i,-i, i, i \frac{1+f}{1-f}\right)
$$

For a $-i$ eigenvector $(a=1)$, we find

$$
\Gamma_{1,-}=\left(i, i,-i \frac{1+f}{1-f},-i\right)
$$

for the same Killing vector $\xi$. For completeness we record the eigenvectors $\left(\xi, \Gamma_{2, \pm}\right)$ of the second generalized almost complex structure $\mathcal{J}_{2}: \Gamma_{2,+}=(-i,-i, i,-i)$ corresponds to the $+i$ eigenvalue and $\Gamma_{2,-}=(i, i, i,-i)$ to the $-i$ eigenvalue.

From (3.12), substituting $\Gamma_{1, \pm}$ as well as the the metric, $B$-field, and Killing vector we get

$$
i c G \Gamma_{1,+}=c(-2 f, 2 f,-4 f, 0), \quad i c B \xi=c(-1+2 f, 1-2 f,-1+2 f, 1-2 f),
$$

where we recall that $c= \pm 1$. We have also identified the 2 -form $B$ in the generalized almost complex structure with the $B$-field. Notice that in order to be able to recover an expression compatible with (3.15), we must take the sum $i c(G \Gamma+B \xi)$, and not the difference of the two terms in (3.18)! The reason for an apparent discrepancy between the two expressions that we have for the moment map, (2.7) and (3.5) lies 
in the identification of the sigma model $B$ field and the 2 -form $B$ that appears in the generalized almost complex structure (3.1). The agreement is restored upon making the identification between minus the sigma model $B$-field and the object by the same name present in (3.1). It is essential that in replacing $B \rightarrow-B$ in (3.1), with $B$ the sigma model $B$-field, we haven't spoiled any of the properties of the generalized Kähler geometry objects.

To complete our argument, we have to make the following assignments for the constants which enter in the one-form $d \sigma: C_{r}=C_{\bar{r}}=C_{\eta}=C_{\bar{\eta}}=i$.

In conclusion, we still find it possible to obtain the moment map from the condition that together with the Killing vector forms a pair $(\xi, i c d \mu)$ which lies in the eigenbundle of the generalized almost complex structure. However, we must exercise caution and interpret the 2 -form $B$ in (3.1) as minus the sigma-model $B$-field. We have also seen that the matching between (3.5) and (2.7) requires making use of the ambiguity in defining the one-form $u$. The exact, $\mathrm{U}(1)$ invariant one-form $d \sigma$ required by the matching between the two moment map definitions led us to a different one-form $d \sigma$ than the one we identified in Section 2.2 by matching $u$ with the gauged sigma model action.

\section{T Duality}

T-duality can be implemented, while preserving the manifest $(2,2)$ supersymmetries of the sigma model, by performing a Legendre transformation of the Kähler potential. This procedure amounts to starting from the gauged sigma model, introducing a Lagrange multiplier that enforces the condition that the gauge field is pure gauge, and eliminating the gauge field from its equation of motion. In terms of the geometric data, by descending to the level of $(1,1)$ superspace, we find that under T-duality, the metric and B-field transform according to the Buscher rules. Let us begin with some review material detailing the execution of T-duality in $(2,2)$ superspace. The simplest example of T-duality involves a non-linear sigma model written in terms of either chiral or twisted chiral superfields with an $\mathrm{U}(1)$ isometry. Under T-duality the chiral multiplets are mapped into twisted anti-chiral and vice-versa. Specifically, we choose a coordinate system such that the isometry is realized by a shift in a particular coordinate. Then the Kähler potential has the form

$$
K=K\left(\bar{\Phi}+\Phi, Z^{a}\right)
$$


where $Z^{a}$ are spectator fields that can be either chiral or twisted chiral. According to the discusion in Section 2.3, the gauged action is obtained by replacing $\bar{\Phi}+\Phi$ with $\bar{\Phi}+\Phi+V$ where $V$ is the usual superfield prepotential for the gauge multiplet. The gauged Kähler potential is

$$
K_{g}=K\left(\bar{\Phi}+\Phi+V, Z^{a}\right)
$$

To construct the duality functional we introduce a Lagrange multiplier that forces the gauge multiplet field strength to vanish:

$$
K_{D}=K_{g}+U(S+i P)+\bar{U}(S-i P) .
$$

Since $(S+i P)=\frac{i}{2} \bar{D}_{+} D_{-} V$ we see that the $U$ and $\bar{U}$ equations of motion force $V$ to be pure gauge, i.e., $V=\Lambda+\bar{\Lambda}$, with $\Lambda$ a chiral superfield. For the next step, by choosing a gauge such that $\Phi+\bar{\Phi}$ have been completely gauged away

$$
K_{g}=K\left(V, Z^{a}\right)
$$

we arrive at the duality functional

$$
K_{D}=K\left(V, Z^{a}\right)-U(S+i P)-\bar{U}(S-i P) .
$$

The original Kähler potential is recovered by integrating out $U$ and $\bar{U}$. The T-dual theory is obtained by integrating out the gauge field. Its equation of motion is

$$
\frac{\partial K}{\partial V}-(\Psi+\bar{\Psi})=0,
$$

where $\Psi=\frac{i}{2} \bar{D}_{+} D_{-} U$ is a twisted anti-chiral superfield. This defines $V=V\left(\Psi+\bar{\Psi}, Z^{a}\right)$. The dual potential

$$
\tilde{K}=K\left(V, Z^{a}\right)-(\Psi+\bar{\Psi}) V
$$

is the Legendre transform of the original potential (4.1).

When one introduces semi-chiral superfields the story becomes somewhat more complicated. In [35], Grisaru et al. gave a detailed discussion of the various descriptions of a $(2,2)$ sigma model, which can be obtained by means of a Legendre transform. Starting with a $(2,2)$ Kähler potential written in terms of semi-chiral superfields $K(X, \bar{X}, Y, \bar{Y})$, one constructs the duality functional

$$
K(r, \bar{r}, s, \bar{s})-X r-\bar{X} \bar{r}-s Y-\bar{s} \bar{Y}
$$


where $r, \bar{r}, s, \bar{s}$ are unconstrained superfields. Depending which fields are integrated out $(X, Y),(r, s),(r, Y),(s, X)$ one finds four equivalent formulations. In the absence of isometries, this amounts to performing a sigma-model coordinate transformation. The authors of [35] investigated the consequences that the existence of an isometry have on the duality functional. For instance if the Kähler potential has a U(1) isometry $K=K(X+\bar{X}, X+\bar{Y}, \bar{X}+Y)$, the duality functional reads $K(r+\bar{r}, \bar{r}+s, r+\bar{s})-$ $(X+\bar{X}-Y-\bar{Y})(r+\bar{r}) / 2+(X-\bar{X}+Y-\bar{Y})(r-\bar{r}) / 2-(r+\bar{s}) Y-(\bar{r}+s) \bar{Y}$. By integrating over $r-\bar{r}$, ultimately leads to expressing $X$ and $Y$ as the sum and difference of a chiral and twisted chiral superfield. In this case, the dual description of the sigma model involves chiral and twisted chiral superfields. The $S U(2) \times U(1)$ WZNW model has two such dual descriptions [33]. The geometry does not change as we pass from one description to the other, but the pair of complex structures does change, from non-commuting complex structures, to commuting ones.

On the other hand, not all the dualities following from (4.8) can be derived from gauging an isometry. The reason is that Lagrange multipliers in (4.8) are semi-chiral superfields. Following the discussion given at the beginning of this section, one would need a gauge multiplet with a semi-chiral field strength, in order to cast the gauged action duality functional (4.5) into (4.8). However, no known $(2,2)$ gauge multiplet contains such a field strength.

Therefore we choose to pursue the construction of the T-dual action of a sigma model with semi-chiral multiplets following the steps which led to (4.5). We add Lagrange multiplier terms to the gauged action as described previously, and construct the duality functional as in [28]. However, a technical difficulty, related to gauge fixing, prevents a straightforward application of this procedure. Let us explain.

The U(1) invariant Kähler potential, which generically takes the form given in (2.13), can be gauged by adding the prepotential $V$ to the appropriate field combinations. The gauged Kähler potential is $K_{g}=K(X+\bar{X}+V, Y+\bar{Y}+V, X+Y+V)$. Because the semi-chiral superfield is not generically reducible in terms of chiral and twisted chiral superfield 4 one cannot completely gauge away $X$ or $Y$, as it was possible for the chiral and/or twisted chiral superfields. Trying to gauge away $X$ we could fix $X\left|=D_{\alpha} X\right|=D^{2} X \mid=0$, where $\mid$ means evaluation with all the Grassmann variables set to zero. Since $X$ has higher order components which are independent of the lower components we realized that we have not gauged away all the $X$ components. The in-

\footnotetext{
${ }^{4}$ We thank Martin Roček for explaining this point to us.
} 
dependent left over components form a $(1,1)$ Weyl spinor superfield. We shall address the resolution to this question in the following section.

\subsection{Dualizing With Chiral and Twisted Chiral Superfields.}

For simplicity we will consider a Kähler potential, parameterized by chiral and twisted chiral superfields, which is strictly invariant under the isometry. The potential is given by (4.1). We begin in the slightly more general setting:

$$
K_{g}=K\left(\bar{\Phi}+\Phi, Z^{a}\right)+\frac{e^{L}-1}{L} V M
$$

The moment map, $M$, is given by $M=i \xi_{\bar{c}} K$, and in this case $\xi_{\bar{c}}=-i \frac{\partial}{\partial \bar{\Phi}}$. To construct the duality functional we add Lagrange multiplier terms that force the superfield strength to vanish. This gives the Lagrangian

$$
K_{D}=K\left(\bar{\Phi}+\Phi, Z^{a}\right)+\frac{e^{L}-1}{L} V M+(\bar{\Psi}+\Psi) V .
$$

The final step is chosing a gauge. Instead of setting $\Phi+\bar{\Phi}=0$, we choose the WessZumino gauge for the prepotential $V$

$$
V\left|=D_{\alpha} V\right|=D^{2} V \mid=0
$$

This gauge choice will allow a better comparison with the semi-chiral case. To see that we do get back the original Lagrangian, we integrate out $\Psi$ and $\bar{\Psi}$. This implies that

$$
V=\bar{\Lambda}+\Lambda
$$

where $\Lambda$ is a chiral superfield. However, consistency with the gauge choice requires that $V=0$ and this give us back the original Kähler potential. To find the dual potential we integrate out $V$. Since $(V)^{3}=0$ in the Wess-Zumino gauge, this allows us to solve for $V$ explicity. We obtain

$$
\begin{aligned}
& V=i \frac{\bar{\Psi}+\Psi+M}{\xi_{\bar{c}} M} \\
& \tilde{K}=K\left(\bar{\Phi}+\Phi, Z^{a}\right)+\frac{i}{2} \frac{(\bar{\Psi}+\Psi+M)^{2}}{\xi_{\bar{c}} M} .
\end{aligned}
$$

The important thing to note here is that consistency of the solution for $\mathrm{V}$ with the gauge fixing conditions require that

$$
V\left|=0=i \frac{\bar{\Psi}+\Psi+M}{\xi_{\bar{c}} M}\right| \Rightarrow(\bar{\Psi}+\Psi)|=-M|
$$


It should be understood that this is a component equation, and not a superfield equation. With this in hand we can show the following;

$$
\frac{\partial^{2} \tilde{K}}{\partial \bar{\Phi} \partial \Phi}\left|=0, \quad \frac{\partial^{2} \tilde{K}}{\partial Z^{a} \partial \Phi}\right|=0, \quad \frac{\partial^{2} \tilde{K}}{\partial \bar{\Psi} \partial \Phi} \mid=-1 .
$$

The implication which follows from these equations is that the contribution of $\Phi \mid$ to the geometry has been replaced by $\Psi \mid$ up to a surface term that comes from the new $B$ field. Let us demonstrate how this works with a simple example, specifically $R \rightarrow \frac{1}{R}$ for one of the cycles on $T^{2}$. The Kähler potential and moment map are:

$$
\begin{aligned}
& K=\frac{R}{2}(\bar{\Phi}+\Phi)^{2} \\
& M=R(\bar{\Phi}+\Phi) .
\end{aligned}
$$

The dual potential is

$$
\tilde{K}=-\frac{1}{2 R}(\bar{\Psi}+\Psi)^{2}-(\bar{\Phi}+\Phi)(\bar{\Psi}+\Psi)
$$

While this looks as though both directions of $T^{2}$ were dualized, one must remember that the real part of $\Psi \mid$ is proportional to $R$ times the real part of $\Phi \mid$. Only the direction parameterized by the imaginary part of $\Phi \mid$ was dualized.

\subsection{Dualizing with semi-chiral superfields}

Now we can give a straightforward extension of the previous discussion to the case when we dualize an isometry of a sigma model parametrized by semi-chiral superfields. We start with equation (2.50), add the Lagrange multipliers enforcing that $V$ is pure gauge, and choose the same gauge Wess-Zumino gauge as in the previous section. The dual potential Kahler potential is:

$$
\tilde{K}=K\left(X, \bar{X}, Y, \bar{Y}, Z^{a}\right)+\frac{i}{2} \frac{(\bar{\Psi}+\Psi+M)^{2}}{\xi_{\bar{c}} M} .
$$

The analogue of (4.15) reads:

$$
\left(\xi_{c}\right)\left(\xi_{\bar{c}}\right) \tilde{K}\left|=0, \quad \frac{\partial\left(i \xi_{\bar{c}} \tilde{K}\right)}{\partial Z^{a}}\right|=0 \quad \frac{\partial\left(i \xi_{\bar{c}} \tilde{K}\right)}{\partial \Psi} \mid=-1
$$

From (4.19) we see that the coordinates in the combination of semi-chiral superfields corresponding to $\xi_{c}$ have been replaced by coordinates in a twisted chiral superfield in 
the dual geometry. This is analogous to what happened in the case of chiral and twisted chiral superfields. It was also expected from gauge fixing considerations, although it was not a propri clear exactly how it would happen. We now have an explicit description of the $\mathrm{T}$ dual of a theory with semi-chiral superfields at the manifest $(2,2)$ sigma model level.

\subsection{An example: T-duality with semi-chiral superfields in flat space}

In this section we try to develop some intuition about the dualization prescription described in the previous section. Given that we perform a duality transformation by gauging away part of a certain combination of semi-chiral superfields, and in doing so we trade it for a twisted chiral superfield, it is not a priori obvious that this is equivalent to the Buscher rules. In particular, we would like to check this in a simple example, namely flat space with a $\mathrm{U}(1)$ isometry.

We start with four-dimensional flat space as our simplest example because one needs both left and right pairs of chiral and anti-chiral superfields in order to be able to eliminate the auxiliary components of the semi-chiral superfields and obtain a sigmamodel action. Therefore we begin with the following $(2,2)$ Kähler potential

$$
K=R(\bar{X}+\bar{Y})(X+Y)-\frac{R}{4}(Y+\bar{Y})^{2}
$$

where $\bar{D}_{+} X=D_{-} Y=0$. By descending to the level of $(1,1)$ superspace using [20], we find the sigma model metric

$$
G=\left(\begin{array}{cccc}
0 & 2 R & R & 0 \\
2 R & 0 & 0 & R \\
R & 0 & 0 & R \\
0 & R & R & 0
\end{array}\right)
$$

where the rows and columns are labelled by $X|, \bar{X}|, \bar{Y}|, Y|$. This gives us the action for the bosonic components

$$
S=\int d^{2} \sigma R\left(\partial^{a} X \partial_{a} \bar{X}+\partial^{a}(\bar{X}+\bar{Y}) \partial_{a}(X+Y)\right)
$$

where for simplicity we denoted by $X$ the bosonic component of the $(1,1)$ superfield $X \mid$. Denoting $Z=X+Y$ we notice that it is inert under the global shift symmetry. 
By performing a diffeomorphism transformation to $(X, \bar{X}, Z, \bar{Z})$, we obtain the metric in canonical form

$$
G=\left(\begin{array}{cccc}
0 & R & 0 & 0 \\
R & 0 & 0 & 0 \\
0 & 0 & 0 & R \\
0 & 0 & R & 0
\end{array}\right)
$$

The T-dual sigma model is obtained from the dual $(2,2)$ Kahler potential given in (4.18). In this particular case, (4.18) reads:

$$
\tilde{K}=R(\bar{X}+\bar{Y})(X+Y)-\frac{R}{4}(Y+\bar{Y})^{2}-\frac{1}{3 R}\left(\psi+\bar{\psi}+R\left(\bar{X}+X+\frac{1}{2}(\bar{Y}+Y)\right)\right)^{2}
$$

and the corresponding T-dual sigma-model metric is equal to:

$$
\frac{9}{2} G=\left(\begin{array}{cccccc}
-4 R & 5 R & 4 R & -5 R & 5 & -4 \\
5 R & -4 R & -5 R & 4 R & -4 & 5 \\
4 R & -5 R & -4 R & 5 R & -5 & 4 \\
-5 R & 4 R & 5 R & -4 R & 4 & -5 \\
5 & -4 & -5 & 4 & -\frac{4}{R} & \frac{14}{R} \\
-4 & 5 & 4 & -5 & \frac{14}{R} & -\frac{4}{R}
\end{array}\right)
$$

where the rows and columns are labelled by $X, \bar{X}, \bar{Y}, Y, \psi, \bar{\psi}$. At first sight this result is puzzling, because we claim that we found the T-dual of a sigma model whose target space is flat four-dimensional space. At the same time, the dual sigma-model involves six fields, and so, apparently the target space is six-dimensional. These two seemingly contradictory statements are reconciled when one takes a closer look at the T-dual metric, and finds that it actually describes a four dimensional subspace. This is obvious when expressing the previous T-dual metric in terms of the following coordinates: $(X, \bar{X}, W=Y-\bar{X}, \bar{W}, \psi, \bar{\psi})$

$$
\frac{9}{2} G=\left(\begin{array}{cccccc}
0 & 0 & 0 & 0 & 0 & 0 \\
0 & 0 & 0 & 0 & 0 & 0 \\
0 & 0 & -4 R & 5 R & 4 & -5 \\
0 & 0 & 5 R & -4 R & -5 & 4 \\
0 & 0 & 4 & -5 & -\frac{4}{R} & \frac{14}{R} \\
0 & 0 & -5 & 4 & \frac{14}{R} & -\frac{4}{R}
\end{array}\right)
$$

where we make the observation that $W=Y-\bar{X}$ is also inert under the global $\mathrm{U}(1)$ action. The final step in getting the metric in its canonical form is to make a coordinate 
transformation to $T=W-\frac{1}{R} \psi$ :

$$
\frac{9}{2} G=\left(\begin{array}{cccccc}
0 & 0 & 0 & 0 & 0 & 0 \\
0 & 0 & 0 & 0 & 0 & 0 \\
0 & 0 & -4 R & 5 R & 0 & 0 \\
0 & 0 & 5 R & -4 R & 0 & 0 \\
0 & 0 & 0 & 0 & 0 & \frac{9}{R} \\
0 & 0 & 0 & 0 & \frac{9}{R} & 0
\end{array}\right) .
$$

This form of the T-dual metric makes it clear that the T-dual geometry is fourdimensional and that the Buscher rules, which in this case amount to $R \rightarrow 1 / R$, are obeyed.

The reason why extracting the T-dual geometry required some work on our part is that the semi-chiral superfields give rise to two $(1,1)$ superfields, one of them being auxiliary. As we gauge an isometry with $(2,2)$ vector superfields, the Lagrange multipliers enforcing the condition that the vector field is pure gauge are twisted chiral superfields (or chiral superfields). To some extent, as we dualize, we exchange the coordinates in a combination of semi-chiral superfields by coordinates in a twisted chiral superfields. However, due to the presence of the auxiliary superfields, even the coordinates which are not directly affected by the duality (like the $Z, \bar{Z}$ coordinates) end up mixing with the dualized coordinate.

\section{Conclusions}

In this paper we have continued the ongoing investigation of the connection between generalized Kähler geometry and two-dimensional $\mathcal{N}=(2,2)$ non-linear sigma models. Specifically, we addressed aspects in the area concerning gauged sigma models with semi-chiral superfields and its relation to various geometric structures on the mathematical side. We have given the form of the gauged action in $(1,1)$ and $(2,2)$ superspace and identified the moment map as well as the one-form needed for the gauging the sigma model, as demonstrated by Hull and Spence in [27]. In two particular cases, namely spaces with almost product structure and the $S U(2) \times U(1)$ WZNW model written in terms of semi-chiral superfields, we have found that the combination of the moment map and Killing vector associated to the isometry $\xi-\sqrt{-1} d \mu^{\xi}$ does lie in the eigenbundle of the generalized almost complex structures, as stipulated by the definitions in the mathematical literature. We leave for a future publication the 
relationship between the physical moment map and the mathematical definition (in the general case). Finally, we have presented a description of T-duality for generic $(2,2)$ sigma models with manifest $(2,2)$ supersymmetry. It is interesting to note that T-duality, as we have described it, introduces a twisted chiral superfield into the sigma model. We could also have described it in such a way that a chiral superfield would be introduced. It would also be interesting to explore the changes which the sigma model geometry undergoes since it appears that the superfield representations for the coordinates get mixed due to T-duality.

\section{Acknowledgments}

We are grateful to L. Anguelova, D. Belov, S.J. Gates, V. Mathai, M. Roček and B. Uribe for comments and suggestions. W.M. thanks the MCTP for hospitality during different stages of this work. WM and LAPZ also thank the KITP for hospitality during the early stages of this work while both were participants of the program "Mathematical Structures in String Theory". This work is partially supported by Department of

Energy under grant DE-FG02-95ER40899 to the University of Michigan and by the National Science Foundation under rant No. PHY99-07949.

\section{References}

[1] B. Zumino, "Supersymmetry And Kahler Manifolds," Phys. Lett. B 87 (1979) 203.

[2] L. Alvarez-Gaume and D. Z. Freedman, "Ricci Flat Kahler Manifolds And Supersymmetry," Phys. Lett. B 94 (1980) 171.

[3] U. Lindstrom and M. Rocek, "Scalar Tensor Duality And N=1, N=2 Nonlinear Sigma Models," Nucl. Phys. B 222, 285 (1983).

[4] M. Rocek and P. K. Townsend, Phys. Lett. B 96, 72 (1980).

[5] T. L. Curtright and D. Z. Freedman, Phys. Lett. B 90, 71 (1980) [Erratum-ibid. B 91, 487 (1980)].

[6] N. J. Hitchin, A. Karlhede, U. Lindstrom and M. Rocek, "Hyperkahler Metrics And Supersymmetry," Commun. Math. Phys. 108 (1987) 535. 
[7] L. Alvarez-Gaume and D. Z. Freedman, "Geometrical Structure And Ultraviolet Finiteness In The Supersymmetric Sigma Model," Commun. Math. Phys. 80, 443 (1981).

[8] S. J. . Gates, C. M. Hull and M. Rocek, "Twisted Multiplets And New Supersymmetric Nonlinear Sigma Models," Nucl. Phys. B 248 (1984) 157.

[9] T. Buscher, U. Lindstrom and M. Rocek, "New Supersymmetric Sigma Models With Wess-Zumino Terms," Phys. Lett. B 202 (1988) 94.

[10] N. Hitchin, "Generalized Calabi-Yau manifolds," Quart. J. Math. Oxford Ser. 54, 281 (2003) [arXiv:math.dg/0209099].

[11] M. Gualtieri, "Generalized Complex Geometry", math.DG/0401221.

[12] H.Bursztyn and M. Crainic, "Dirac structures, moment maps and quasi-Poisson manifolds," math.DG/0310445

[13] M. Crainic, "Generalized complex structures and Lie brackets," math.DG/0412097

[14] Y. Lin and S. Tolman, "Symmetries in generalized Kähler geometry," math.DG/0509069

[15] Y. Lin and S. Tolman, "Reduction of twisted generalized Kähler structure," math.DG/0510010

[16] S. Hu, "Hamiltonian symmetries and reduction in generalized geometry," math.DG/0509060.

[17] H. Bursztyn, G. Cavalcanti and M. Gualtieri, "Reduction of Courant algebroids and generalized complex structures," math.DG/0509640

[18] U. Lindstrom, R. Minasian, A. Tomasiello and M. Zabzine, "Generalized complex manifolds and supersymmetry," Commun. Math. Phys. 257, 235 (2005) [arXiv:hep-th/0405085].

[19] U. Lindstrom, M. Rocek, R. von Unge and M. Zabzine, "Generalized Kaehler geometry and manifest $\mathrm{N}=(2,2)$ supersymmetric nonlinear sigma-models," JHEP 0507 (2005) 067 [arXiv:hep-th/0411186]. 
[20] U. Lindstrom, M. Rocek, R. von Unge and M. Zabzine, "Generalized Kaehler manifolds and off-shell supersymmetry," arXiv:hep-th/0512164.

[21] A. Bredthauer, U. Lindstrom and J. Persson, "First-order supersymmetric sigma models and target space geometry," JHEP 0601, 144 (2006) [arXiv:hepth/0508228].

[22] A. Bredthauer, U. Lindstrom, J. Persson and M. Zabzine, "Generalized Kaehler geometry from supersymmetric sigma models," arXiv:hep-th/0603130.

[23] N. Hitchin, "Instantons, Poisson structures and generalized Kaehler geometry," arXiv:math.dg/0503432.

[24] C. M. Hull, G. Papadopoulos and B. J. Spence, "Gauge symmetries for (p,q) supersymmetric sigma models," Nucl. Phys. B 363, 593 (1991).

[25] C. M. Hull, A. Karlhede, U. Lindstrom and M. Rocek, "Nonlinear Sigma Models And Their Gauging In And Out Of Superspace," Nucl. Phys. B 266, 1 (1986).

[26] J. Bagger and E. Witten, "The Gauge Invariant Supersymmetric Nonlinear Sigma Model," Phys. Lett. B 118, 103 (1982).

[27] C. M. Hull and B. J. Spence, "The gauged nonlinear sigma model with WessZumino term," Phys. Lett. B 232, 204 (1989).

[28] M. Rocek and E. P. Verlinde, "Duality, quotients, and currents," Nucl. Phys. B 373, 630 (1992) [arXiv:hep-th/9110053].

[29] C. M. Hull and B. J. Spence, "The Geometry of the gauged sigma model with Wess-Zumino term," Nucl. Phys. B 353, 379 (1991).

[30] S. J. J. Gates, "Vector multiplets and the phases of $\mathrm{N}=2$ theories in 2-D: Through the looking glass," Phys. Lett. B 352, 43 (1995) [arXiv:hep-th/9412222].

[31] L. Alvarez-Gaume and D. Z. Freedman, "Potentials For The Supersymmetric Nonlinear Sigma Model," Commun. Math. Phys. 91, 87 (1983).

[32] A. N. Jourjine, "The Effective Potential In Extended Supersymmetric Nonlinear Sigma Annals Phys. 157, 489 (1984). 
[33] I. T. Ivanov, B. b. Kim and M. Rocek, "Complex structures, duality and WZW models in extended superspace," Phys. Lett. B 343, 133 (1995) [arXiv:hepth/9406063].

[34] A. Sevrin and J. Troost, "Off-shell formulation of N $=2$ non-linear sigma-models," Nucl. Phys. B 492 (1997) 623 [arXiv:hep-th/9610102].

A. Sevrin and J. Troost, "The geometry of supersymmetric sigma-models," arXiv:hep-th/9610103.

[35] M. T. Grisaru, M. Massar, A. Sevrin and J. Troost, "Some aspects of N $=(2,2)$, D = 2 supersymmetry," Fortsch. Phys. 47, 301 (1999) [arXiv:hep-th/9801080].

[36] S. Hu, "Reduction and duality in generalized geometry," math.DG/0512634 